\title{
Effects of mannuronic acid (M2000) on gene expression profile of signal transducer and activator of transcription proteins (STATs) in rheumatoid arthritis patients
}

\author{
N.A.G. Gaafar ${ }^{1,2}$, M. Aslani' ${ }^{1}$, Z. Aghazadeh1', S.S. Mortazavi-Jahromi', \\ A. Razavi', A. Mirshafiey ${ }^{1,3}$ \\ ${ }^{1}$ Department of Immunology, School of Public Health, Tehran University of Medical Sciences, Tehran, Iran; \\ ${ }^{2}$ National Public Health Laboratory, Khartoum, Sudan; ${ }^{3}$ Research Centre for Immunodeficiencies,
Children's Medical Centre, Tehran University of Medical Sciences, Tehran, Iran \\ ${ }^{2}$ National Public Health Laboratory, Khartoum, Sudan; ${ }^{3}$ Research Centre for Immunodeficiencies,
Children's Medical Centre, Tehran University of Medical Sciences, Tehran, Iran
}

\begin{abstract}
SUMMARY
Rheumatoid arthritis (RA), a form of inflammatory arthritis, is a chronic joint disease characterized by pain and inflammation that affects $0.5 \%$ to $1 \%$ of the population worldwide. The safety, efficacy, tolerability, and potency of $\beta$-D-mannuronic acid (M2000) as a novel NSAID with immunosuppressive property has been reported by several in vitro studies, experimental models and clinical trials phase I/II and III in ankylosing spondylitis and rheumatoid arthritis (RA) patients This research is designed to study the therapeutic efficacy of oral administration of mannuronic acid in RA patients who had inadequate response to conventional drugs and to assess the effect of this drug on gene expression of the signal transducer and activator of transcription (STATs) protein (STAT1, STAT3, STAT4, and STAT6). The study has included 15 RA patients who had an insufficient response to the conventional therapy. The oral dose of mannuronic acid was $1000 \mathrm{mg}$ divided into two $500 \mathrm{mg}$ doses per day for 3 months as an addition to conventional therapy. There were 15 healthy volunteer in the control group. Blood samples were collected from both groups, once from healthy controls and twice from RA patients before and after treatment by M2000. The peripheral blood mononuclear cells (PBMCs) were isolated to assess the gene expression level of STAT1, STAT3, STAT4, and STAT6 using the real-time PCR method. Results obtained in this study demonstrated a significant difference in the gene expression level of STAT1 between healthy controls and patients before treatment as well as a significant reduction in RA patients after treatment compared with the level before treatment. In addition, the gene expression level of STAT3 and STAT4 showed a significant reduction in RA patients after treatment compared to patients before treatment, while there was no significant difference between RA patients before treatment and the healthy control group for both molecules. On the other hand, there was no change in the gene expression level of STAT6 among all groups. The outcomes of this study confirmed that $\beta$-D-mannuronic acid (M2000) has the ability to control the levels of STAT1, STAT3 and STAT4 in RA patients, and might be beneficial in the management and therapy of RA.
\end{abstract}

Key words: Mannuronic acid M2000; STAT1; STAT3; STAT4; STAT6; arthritis; rheumatoid; gene expression.

Reumatismo, 2020; 72 (2): 93-102

\section{INTRODUCTION}

heumatoid arthritis (RA) is a widespread common autoimmune disease associated with progressive disability, systemic complications, early death, and socioeconomic cost (1). RA is a persistent autoimmune inflammatory synovitis that affects about $1 \%$ of the population and contributes to functional disability (2) The etiology of RA has not yet been identified. However, autoimmune mechanisms play a key role in the pathogenesis of the disease (3). Chronic inflammation in RA patients causes a synovial proliferation causing cartilage and bone absorption (4). RA is a persistent autoimmune inflammatory disease leading to progressive joint degenera- $\overline{\text { Corresponding author: }}$ Abbas Mirshafiey Department of Immunology, School of Public Health, Tehran University of Medical Sciences, Box: 14155-6446, Tehran, Iran E-mail: mirshafiey@tums.ac.ir 
tion, disability, and elevated risk of cardiovascular complications, that represent the major cause of mortality among RA patients (5). The etiopathogenesis of RA is multifactorial and not fully understood, like in most autoimmune diseases. The autoimmune process leads to the dysregulation of cytokine synthesis, disturbances in the migration of immunocompetent cells and abnormal apoptosis. Up to date, the mechanisms through which inflammatory mediators, specifically cytokines, influence effector cells have not been fully studied. One of the transduction pathways that was found to have this influence is the Janus tyrosine kinase/signal transducer and activator of transcription (JAK/STAT) pathway. So far, seven homologous proteins of the STAT family have been identified in mammalian cells: STAT1, $-2,-3,-4$, $-5 \mathrm{~A},-5 \mathrm{~B}$ and -6 . A single STAT type can be stimulated by many different ligands, but in general cytokines preferentially use a specific transduction pathway, e.g., INF $\gamma$ usually selects STAT1 (6). When a cytokine is bound to a cell membrane receptor, the associated JAK tyrosine kinase is activated and the process of phosphorylation takes place. The molecules bind with subunits of cytoplasmic STAT proteins, which undergo dimerization. This complex is then translocated into the nucleus via importing. In the nucleus, STAT is released and binds with a DNA fragment responsible for the expression of a specific arrangement of genes (7). Previous studies have investigated the deregulation of the JAK/STAT pathway in RA. Elevated expression of the STAT1, STAT-3 and STAT-4 and proteins were found during active RA synovitis (6). Up-regulated levels of STAT3 mRNA in mononuclear cells from peripheral blood and synovial fluid, and elevated STAT1 expression in the synovial fluid have been observed in active RA (8). STAT3 has been found to be responsible for joint degeneration in RA (9). STAT1 controls cell growth proliferation, apoptosis, and functions of the immune system. Polymorphisms of STAT1 have been related to an increased risk of malignancy (10). In humans with autosomal-recessive deficiency of STAT1, this deficiency leads to the progression of a primary immunodeficiency syndrome characterized by susceptibility to viral infections and bacterial pathogens (11). STAT1 has a role in inhibiting certain autoimmune disorders. Conversely, STAT1 mediates anti-proliferative and proapoptotic effects of IFNs, suggesting that STAT1 has also the potential for suppressing inflammation (12).

STAT3 is a pleiotropic transcription factor, which can play a role in the signal of different cytokines involving IL-6, IL-10, and other gp130 cytokines. Additionally, it could be included in immune and somatic cell abnormalities $(12,11)$. Recent research demonstrated that STAT3 plays an important role in inducing and maintaining a procarcinogenic inflammatory microenvironment. Therefore, STAT3 is believed to contribute to promoting oncogenesis $(13,14)$. In general, STAT3 has a complex role, as it can have different effects on various cells, depending on the cell type and activation status (15). Contrary to other STATs, that are expressed on a wide range of cell types, STAT4 is predominantly expressed by immune cells and the testis (16). STAT4 is activated by type I IFN in humans through interaction with STAT2 $(17,18)$. STAT4 is an important transcription factor for the biological function of several immune cells, such as macrophages, mast cells, natural killer cells, dendritic cells, T helper (Th) cells, regulatory $\mathrm{T}$ cells, follicular helper $\mathrm{T}$ cells, CD8+T cell and B cells (19-22). STAT4 can affect the activities of these cells that play a role in the pathogenesis of autoimmune diseases, such as systemic lupus erythematosus, RA, inflammatory bowel disease, multiple sclerosis, type 1 diabetes, systemic sclerosis, psoriasis and experimental autoimmunemyocarditis (19). By activating IL-12, STAT4 has an important role in the generation and proliferation of Th1 cells and is essential for the progression of Th17 cells $(23,24)$.

Up to $40 \%$ of patients with rheumatoid arthritis (RA) are insufficient responders (IR) to conventional disease-modifying antirheumatic drugs (DMARDs) or tumour necrosis factor $\alpha$ inhibitor (TNFi) biological 
agents $(25,26)$. Patients who are intolerant and/or show an inadequate response to traditional DMARDs (DMARD-IR) are often treated with a biologic agent (27). For DMARD-IR patients, biologics are usually combined with traditional DMARDs, primarily MTX, but some biologics and also tofacitinib have shown to be effective as monotherapy as well (28-30). Adverse events (primarily gastrointestinal symptoms, respiratory symptoms, and hepatotoxicity) are the main reasons $(>75 \%)$ for MTX withdrawal (31).

A systematic review reported two pivotal, international, double-blind randomized controlled trials, sponsored by the manufacturers, BEACON and BUILD, which both enrolled adult patients inadequately controlled on bDMARDs (TNF inhibitors) and, in the latter, patients were inadequately controlled on cDMARDs. Both studies had a 24-week double-blind treatment period in which baricitinib $2 \mathrm{mg}$ and baricitinib $4 \mathrm{mg}$ were compared with placebo. The primary outcome in each study was the proportion of patients achieving an American College of Rheumatology (ACR) improvement criteria of at least 20\% (ACR20) response at 12 weeks. Key secondary outcomes that were accounted for multiplicity included health-related quality of life (HRQoL) on the Health Assessment Questionnaire-Disability Index (HAQ-DI), the Disease Activity Scale-28 and high-sensitivity C-reactive protein (DAS28-hs-CRP), and the Simplified Disease Activity Index (SDAI).

The primary outcome for both BUILD and BEACON was the proportion of patients achieving ACR20 at week 12. In both BEACON and BUILD (48.9\% of baricitinib patients and $27.3 \%$ of placebo patients) and BUILD (65.9\% versus $39.5 \%)$, more participants in the baricitinib group than in the placebo group achieved ACR20, and these differences were statistically significant between the groups. The proportion of patients achieving ACR20 at 24 weeks was higher with baricitinib than with placebo in BEACON (44.8\% versus $27.3 \%)$ and BUILD (61.1\% versus $42.1 \%)$. Interesting subgroup analyses were per- formed on the primary outcome (ACR20 responses at week 12) based on the prior reason for failure on bDMARDs (lack of efficacy, adverse event, etc.) and for the number of previous bDMARDs in BEACON. Results for the subgroup which had a lack of efficacy were $49.1 \%$ for the baricitinib group versus $27.1 \%$ for the placebo group. In the other subgroup, there were only three patients in both groups. Therefore, it is difficult to understand why $50 \%$ responded in the baricitinib group and $100 \%$ in the placebo group. In both studies, baricitinib reduced (improved) the HealthRelated Quality of Life (HAQ-DI), EQ-5D Health State Index/Self-Perceived Health score, DAS28-hs-CRP and MCID on the SDAI from baseline to week 12 in RA patients compared with placebo (32).

The $\beta$-D-mannuronic acid (M2000) (DE102016113018.4), a novel NSAID with immunosuppressive property, is a safe agent with no toxicity for the GI tract and renal function (33). It has shown therapeutic benefits with the greatest tolerability, safety, and efficacy in various animal disease models, such as experimental autoimmune encephalomyelitis (EAE), adjuvantinduced arthritis (AIA), acute glomerulonephritis and nephrotic syndrome (34-37). The treatment with M2000 in combination with the conventional therapy demonstrated considerably greater efficacy along with a high safety profile compared to patients treated with the conventional therapy. Therefore, M2000 might be recommended as an appropriate choice in the remission of RA (38). The multinational phase III clinical trial showed that mannuronic acid is an effective and safe drug and is generally well-tolerated in patients with RA (39).

\section{MATERIALS AND METHODS}

\section{Clinical characterization of patients and controls}

15 rheumatoid arthritis patients with active disease were enrolled in this study. $80 \%$ were females and $20 \%$ were males. They were suffering from inadequate response to conventional drugs. The conventional therapy included DMARDs [Methotrexate 
(MTX) 15-20 mg/week, Sulfasalazine (SSZ) 500-1000 mg/day and hydroxychloroquine (HCQ) $400 \mathrm{mg} /$ day], corticosteroids (Prednisolone (PRD) 5-15 mg/day) and NSAIDs. As to the inclusion criteria, the RA patients were to use the drugs at least for 6 months before this study. The mean age was $52.33 \pm 1.65$ and the mean disease duration was $8.08 \pm 1.60$ years. The selected criteria for evaluating the immunological parameters of RA patients were based on the American College of Rheumatology recommendations, which considered DAS $\geq 3$ in their clinical evaluation. The dose of mannuronic acid was $1000 \mathrm{mg}$ per day divided by 2 , then $500 \mathrm{mg}$ in each capsule for three months, which was simultaneously administered with conventional drugs. Before starting our investigation, all the patients were informed and asked to sign an informed consent. Then, the patients received the follow-up appointments at baseline, 4 weeks and 12 weeks at the Department of Rheumatology in Loghman Hakim hospital Tehran, Iran, and the Division of Rheumatology Research, Rheumatism Center. Further follow-up was done by telephone in order to assess the adverse events of mannuronic acid. In addition, 15 individuals were enrolled as a healthy control group.

\section{Ethical statement}

The ethical approval with reference code number IR.TUMS.VCR.REC.1395.6212016-09-14 was obtained from the Ethical Committee of Tehran University of Medical Sciences (TUMS) for this research study. A written and signed informed consent was obtained from all participants in the research. The research was conducted under the guidelines established by the American College of Rheumatology (ACR) and the Helsinki manifesto and its later amendments or comparable ethical standards.

\section{$\beta$-D-mannuronic acid preparation and intake}

The patented small molecule (DE-102016 113018.4) $\beta$-D-mannuronic acid (M2000) with molecular formula $\mathrm{C} 6 \mathrm{H} 10 \mathrm{O} 7$ was synthesized from alginic acid sodium salt (Sigma-Aldrich, St. Louis, MO, USA) based on a modified method of the acid hydrolysis tested (32). The purity of M2000 was approved by characterizing the hydrolytic products using Fourier transform infrared (FTIR) spectroscopy and carbon-13 nuclear magnetic resonance (13CNMR) spectroscopy for asserting its molecular weight $(194.139 \mathrm{~g} / \mathrm{mol})$ and exact/monoisotopic mass. The preclinical studies showed that M2000 is an anti-inflammatory agent with high biocompatibility and no toxicity on functions of kidneys and the GI tract (40-43).

\section{Sample collection}

Blood samples were collected once from 15 healthy volunteers and twice from 15 RA patients before and after treatment. Afterward, the peripheral blood mononuclear cells (PBMCs) were isolated using FicollPaque (Amersham Pharmacia Biotech, Uppsala, Sweden) and stored at $-70^{\circ} \mathrm{C}$. The entire amount of RNA was extracted from $2 \times 10^{6}-5 \times 10^{6}$ cells using GeneAll ${ }^{\circledR}$ HybridRTM kits (Qiagen, Valencia, CA, USA) according to manufacturer's guidelines and placed into $50 \mu \mathrm{L}$ of RNase-free water. The NanoDrop 2000 UV-Vis Spectrophotometer (Thermo Fisher Scientific, Waltham, MA, USA) was used to calculate the concentration of total RNA. It was then concentrated or diluted to a concentration of $<300 \mathrm{ng} / \mu \mathrm{L}$ for cDNA synthesis.

\section{Quantitative real-time polymerase chain reaction}

Reverse-transcribed random SolisBio Dyne primers were used for real-time PCR based on cDNA Synthesis Kit protocol (Yekta E Takara Co., Ltd., Dalian, China). Quantitative real-time PCR was performed using $5 \mathrm{X}$ HOT FIREPol ${ }^{\circledR}$ EVAGreen ${ }^{\circledR} \mathrm{q}$ PCR Mix Plus (ROX) with a specified primer (Table I) according to the available instructions. The analysis of STAT1, STAT3, STAT4, and STAT6 was fulfilled by ABI StepOnePlus ${ }^{\text {TM }}$ Real-Time PCR system (Applied Biosystems, Foster City, CA, USA). The relative quantities of selected genes mRNA were compared against one internal control, 
Table I - The designed forward and reverse primers.

\begin{tabular}{|c|c|c|}
\hline Gene Name & Forward primer & Reverse primer \\
\hline STAT1 & ACCTAACGTGCTGTGCGTAG & GAGACATCCTGCCACCTTGT \\
\hline STA3 & TCCTGAAGCTGACCCAGGTA & AGGTGAGGGACTCAAACTGC \\
\hline STAT4 & CATCTCAACAATCCGAAGTGATCA & GTCAGAGTTATCCTGTCATTCAGCAG \\
\hline STAT6 & ACTCCAGGAGAGGTGTGAAAG & GAAGCAACTGGTGACGAGG \\
\hline GAPDH & GAGAAGGCTGGGGCTCATT & TAAGCAGTTGGTGGTGCAGG \\
\hline
\end{tabular}

GAPDH Mrna, which was calculated by considering the $\triangle \mathrm{CT}$ method using an amplification plot (fluorescence signal vs cycle number). The difference $(\Delta \mathrm{CT})$ between the mean values in the duplicate samples of target genes and GAPDH mRNA was calculated. Afterward, the variation in $(\Delta \Delta \mathrm{CT})$ values of the gene expression of selected molecules among the groups was measured and reported as $2-\Delta \Delta \mathrm{CT}$.

\section{Statistical analysis}

All statistical analyses were carried out by SPSS software (24.0; IBM Corporation North Castle, Armonk, NY, USA). All data were expressed as means, standard deviations and $\mathrm{p}<0.05$ was considered statistically significant. The KolmogorovSmirnov test was used to check the normality of all data. The Analysis of Variance (ANOVA) test was used to compare the quantitative variables between the groups and the Newman-Keuls test to determine significant differences in the gene expression level between the untreated and treated groups. Statistical significance was classified as $* p \leq 0.05, * * p \leq 0.01$ or $* * * p \leq 0.001$. A $p$-value of $\leq 0.05$ was considered statistically significant.

\section{RESULTS}

\section{Patient's response to treatment}

After 3 months of mannuronic acid therapy, the medical reports showed an improvement in the clinical parameters of patients. At the end of the treatment, a significant decrease was observed in the level of Disease Activity Score for the 28-joint count (DAS28), tender joints count, swollen joints, patient's assessment and erythrocyte sedimentation rate (ESR), while there was
Table II - Clinical characterizations of patients before and after M2000 therapy.

\begin{tabular}{|l|c|c|c|}
\hline Index & Before treatment & After treatment & P-value \\
\hline DAS28 & $4.46 \pm 0.23$ & $2.83 \pm 0.11$ & 0.001 \\
\hline Tender joint count & $4.00 \pm 0.56$ & $1.00 \pm 0.27$ & 0.001 \\
\hline Swollen joint count & $2.33 \pm 0.64$ & $0.50 \pm 0.19$ & 0.011 \\
\hline Patient assessment of pain & $66.67 \pm 3.95$ & $40.00 \pm 4.76$ & 0.005 \\
\hline ESR & $22.33 \pm 3.83$ & $14.08 \pm 2.65$ & 0.016 \\
\hline CRP & $41.7 \%$ (Positive) & $33.3 \%$ (Positive) & 1.000 \\
\hline RF & $58.3 \%$ (Positive) & $50 \%$ (Positive) & 1.000 \\
\hline
\end{tabular}

DAS28, Disease Activity Score of 28-joint, ESR, erythrocyte sedimentation rate; RF, rheumatoid factor; CRP, C-reactive protein.

no significant difference in the level of rheumatoid factor $(\mathrm{RF})$ and $\mathrm{C}$-reactive protein (CRP) (Table II).

\section{The effects of $\beta$-D-mannuronic acid on STAT1 gene expression}

Our findings demonstrated a significant difference in the gene expression level of STAT1 in the healthy control group compared to RA patients before treatment, as well as a significant difference between RA patients before and after a 12-week therapy with M2000 (Figure 1). Consequently, STAT1 as an inflammatory marker appeared significantly decreased following the treatment with M2000 in RA patients $(\mathrm{p} \leq 0.01)$.

\section{The effects of $\beta$-D-mannuronic acid on STAT3 gene expression}

In the gene expression level of STAT3, there was no significant difference between the healthy control group and RA patients before the M2000 therapy. Vice versa, there was a statistically significant difference in the gene expression level of STAT3 in the comparison between RA patients group be- 


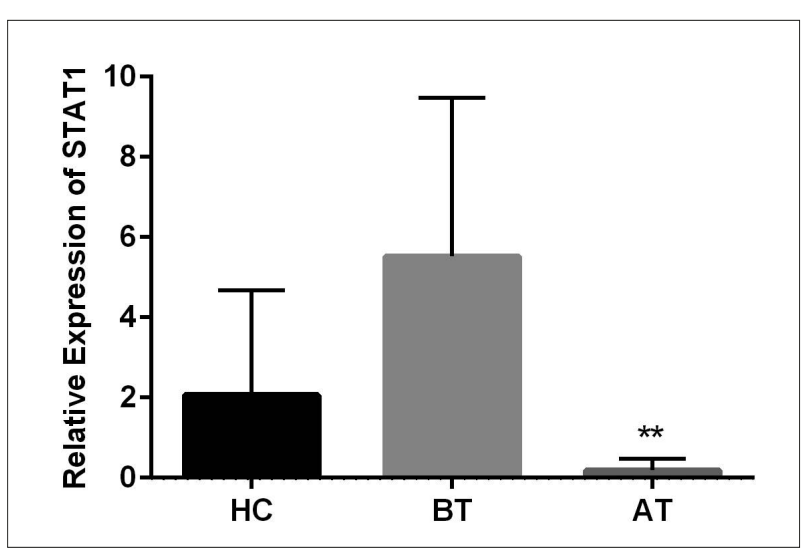

Figure 1 - Effects of $\beta$-D-mannuronic acid on STAT1 gene expression. $\mathrm{HC}$, healthy control; BT, before treatment; AT, after treatment. The outcomes are shown as mean $\pm S E M$. $p$-value $\leq 0.05$ was considered statistically significant. A significant reduction can be seen compared to before treatment: ${ }^{* *} p \leq 0.01$.

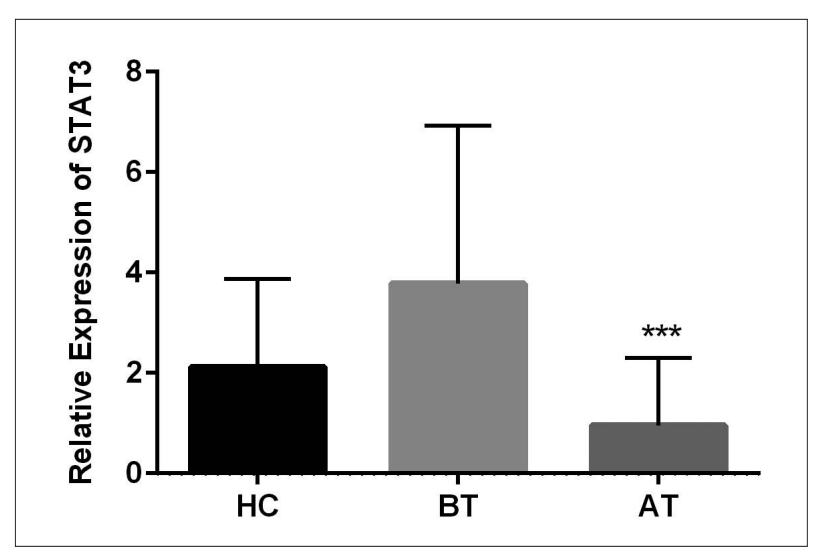

Figure 2 - Effects of $\beta$-D-mannuronic acid on STAT3 gene expression. $\mathrm{HC}$, healthy control; BT, before treatment; $\mathrm{AT}$, after treatment. The outcomes are shown as mean \pm SEM. $p$-value $\leq 0.05$ was considered statistically significant. A significant reduction can be seen compared to before treatment: ${ }^{* \star *} \mathrm{p} \leq 0.001$. fore and after treatment with mannuronic acid for 12 weeks $(\mathrm{p} \leq 0.001)$ (Figure 2).

\section{The effects of $\beta-D$-mannuronic acid on STAT4 gene expression}

The gene expression level of STAT4 showed a statistically significant difference in the comparison between RA patients before and after treatment with M2000 for 3 months $(\mathrm{p} \leq 0.00)$. In addition, the clinical parameters reduced following the treat- ment. On the other side the difference in the gene expression level of STAT4 between the healthy control group and the RA patients before treatment was not significant (Figure 3).

\section{The effects of $\beta-D$ - mannuronic acid on STAT6 gene expression}

In contrast to previous groups of STATs, there was no significant difference in the gene expression level of STAT6 either in

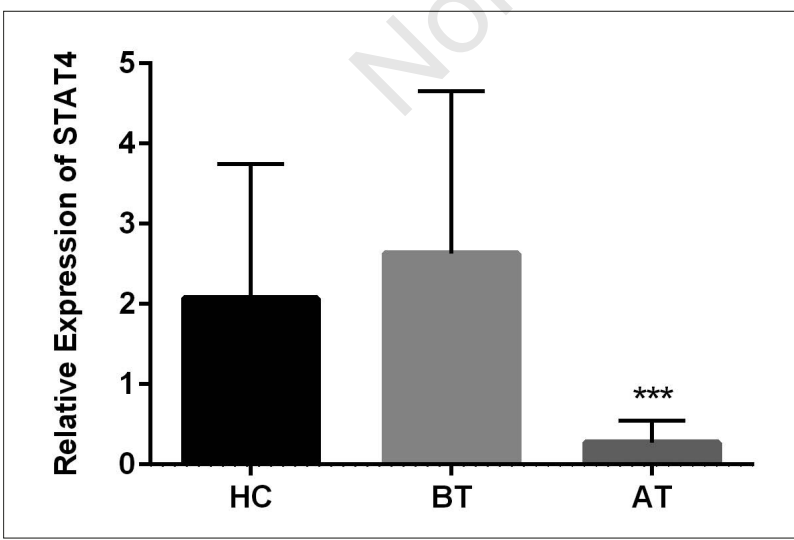

Figure 3 - Effects of $\beta$-D-mannuronic acid on STAT4 gene expression. $\mathrm{HC}$, healthy control; $\mathrm{BT}$, before treatment; $\mathrm{AT}$, after treatment. The outcomes show mean \pm SEM. $p$-value $\leq 0.05$ was considered statistically significant. A significant reduction can be seen compared to the value before treatment: ${ }^{* * *} p \leq 0.001$.

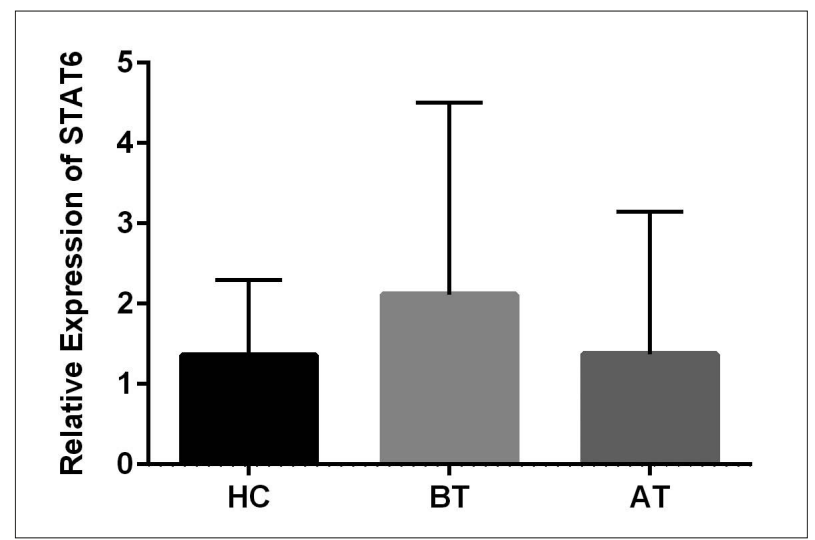

Figure 4 - Effects of $\beta$-D-mannuronic acid on STAT6 gene expression. $\mathrm{HC}$, healthy control; BT, before treatment; $A T$, after treatment. The outcomes show mean $\pm S E M$. $p$-value $\leq 0.05$ was considered statistically significant. No significant difference was identified in the gene expression level of STAT6 between all groups: $p \leq 0.093$. 
healthy controls or in RA patients before and after the treatment with $\beta$-D-mannuronic acid $(\mathrm{p} \leq 0.093)$ (Figure 4).

\section{DISCUSSION}

Rheumatoid arthritis (RA) is a chronic autoimmune disorder that affects $\sim 1-2 \%$ of the population worldwide. RA is characterized by inflammation, autoantibody production, cartilage and bone damage, and synovial hyperplasia. Inflammation promotes the synthesis of pro-inflammatory cytokines both systemically and in the joints, such as tumor necrosis factor-alpha and interleukin-6, which play a significant role in joint and other organ damage in this disease. Considering the role of the signal transducer and activator of transcription factors (STATs) in the signalling of these cytokines, these proteins may participate in the pathogenesis of RA. The expression and activity of STATs can contribute to the onset, progression, and virulence of RA. All STAT family members (STAT1, STAT2, STAT3, STAT4, STAT5a, STAT5b, and STAT6) have been related to autoimmune diseases, as highlighted in numerous research works (15). Despite several existing treatments and recent progress in disease therapy, remission rates and morbidity remain a critical concern for RA patients (44). Given the irreversible devastating damages caused by joint inflammation and the avalability of effective disease-modifying drugs, it is widely recognized that earlier treatment is needed for more effective management of RA. There is also some evidence that early intervention can potentially be a curative treatment (45). Given the availability of various alternative biologic treatments and other novels diseasemodifying antirheumatic drugs (DMARDs) for the treatment of patients with RA, it is increasingly challenging for clinicians to identify the optimal treatment. The biologic therapies are usually combined with traditional DMARDs, primarily methotrexate (MTX), but some biologics and tofacitinib have shown to be effective also as a monotherapy (46).

According to BEACON and BUILD, ba- ricitinib is reported to improve clinical parameters in RA patients compared with placebo at 12 weeks, yet it was associated with serious adverse effects, such as upper respiratory tract infection, occurring in $9 \%$ of baricitinib patients and $5 \%$ of placebo patients in BEACON and in $6 \%$ of baricitinib and $8 \%$ of placebo patients in BUILD. Herpes zoster as a serious adverse event occurred in $1 \%$ of patients in each group in BEACON and $2 \%$ of baricitinib patients versus no placebo patients in BUILD. In addition, it also caused other notable events, including malignancies, thrombotic events, dyslipidemia, and elevations in hepatic enzymes. In addition, there were no clear differences between groups within BEACON and BUILD. There was a higher risk of elevated platelet counts with baricitinib versus placebo in BUILD (19\% versus 5\%); however, there was a much smaller difference between groups in BEACON ( $18 \%$ versus $14 \%$ ). Low neutrophil counts were seen in $6 \%$ of baricitinib patients and $2 \%$ of placebo patients in BEACON and $8 \%$ of baricitinib patients versus $4 \%$ of placebo patients in BUILD. No patients had gastrointestinal perforation in either studies (32). Mannuronic acid as a novel and natural anti-inflammatory drug, which proved its safety, efficacy, tolerability, and potency in in-vivo and in-vitro tests and clinical trials in phase $\mathrm{I} / \mathrm{II}$ in ankylosing spondylitis and in phase I/II and III in RA patients has been used in this study for 3 months in RA patients in order to evaluate its effect on STATs molecules. The results showed a significant response in the proposed gene expression as well as in clinical and para-clinical results. The previous clinical trial in RA patients evaluated different genes, but, in this study, we investigated the effect of this new drug on STATs which play an important role in the pathogenicity of this disease. In the synovia of RA patients and in experimental arthritis, STAT1 expression is increased and both STAT1 and STAT3 are in an activated state (4749). STAT1 as an inflammatory factor was significantly different between the healthy control group and RA patients, even before the treatment, showing the importance of 
this molecule in the pathogenicity of RA. In addition, the difference between patients before treatment and after treatment with mannuronic acid over 3 months was highly significant. Also, the gene expression of STAT1 was decreased following the treatment, thus proving that this drug is promising in controlling STAT1 transcription factors, which participate in regulating Th17 differentiation and controlling cell infiltration in inflamed joints (50). Not only did M2000 have an effect on STAT1, but it also had a significant effect on the gene expression level of STAT3 and STAT4 in RA patients after treatment compared to patients before treatment, as it reduced the gene expression of both molecules, which were elevated during the disease. These data indicated that this drug is effective in controlling both transcription factors in RA patients and suitable for the treatment of the disease. On the contrary, there was no significant difference in the gene expression levels of both molecules in the healthy control group compared to RA patients before treatment. This confirms again the efficacy of this drug on controlling both transcription factors, which participate in the pathogenicity in RA patients and reducing the level of gene expression of both molecules, which increased during the disease. On the other hand, this drug has not shown a significant effect on the gene expression level of STAT6 among the groups.

In conclusion our findings confirm the results of previous studies of mannuronic acid and its efficacy in different autoimmune diseases. This drug showed high efficacy in controlling the gene expression level of the transcription factors STST1, STAT3 and STAT4, which have a significant role in the pathogenicity of RA. This drug which has shown its safety, efficacy, tolerability, and potency in different studies, has proven effective also in the treatment of RA patients and could be considered as a drug of choice in the near future.

\section{Data availability}

Data utilized to support the findings of this study are available from the corresponding author upon request.

\section{Conflict of interest}

The authors declare that they do not have any conflicts of interest.

\section{REFERENCES}

1. Aletaha D, Smolen JS. Diagnosis and management of rheumatoid arthritis: a review. JAMA. 2018; 320: 1360.

2. Guo Q, Wang Y, Xu D, et al. Rheumatoid arthritis: pathological mechanisms and modern pharmacologic therapies. Bone Res. 2018; 27: 15.

3. Choy E. Understanding the dynamics: pathways involved in the pathogenesis of rheumatoid arthritis. Rheumatology. 2012; 51: v3-11.

4. Jimenez-Boj E, Redlich K, Türk B, et al. Interaction between synovial inflammatory tissue and bone marrow in rheumatoid arthritis. J Immunol. 2005; 175: 2579-88.

5. Totoson P, Maguin-Gaté K, Prati C, Demougeot C. Mechanisms of endothelial dysfunction in rheumatoidarthritis: lessons from animal studies. Arthritis Res Ther. 2014; 16: 202.

6. Ivashkiv LB, Hu X. The JAK/STAT pathway in rheumatoid arthritis: pathogenic orprotective? Arthritis Rheum. 2003; 48: 2092-6.

7. Stark GR, Darnell JE. The JAK-STAT pathway at twenty. Immunity. 2012; 36: 503-14.

8. Isomäki P, Junttila I, Vidqvist KL, et al. The activity of JAK-STAT pathways in rheumatoid arthritis: constitutive activation of STAT3 correlates with interleukin-6 levels. Rheumatology. 2015; 54: 1103-13.

9. Mori T, Miyamoto T, Yoshida H, et al IL-1 $\beta$ and TNF $\alpha$-initiated IL-6-STAT3 pathway is critical in mediating inflammatory cytokines and RANKL expression in inflammatory arthritis. Int Immunol. 2011; 23: 701-12.

10. Butterbach K, Beckmann L, de Sanjosé S, et al. Association of JAK-STAT pathway related genes with lymphoma risk: results of a european case-control study (EpiLymph). $\mathrm{Br} \mathrm{J}$ Haematol. 2011; 153: 318-33.

11. Hambleton S, Goodbourn S, Young DF, et al. STAT2 defciency and susceptibility to viral illness in humans. Proc Natl Acad Sci USA. 2013; 110: 3053-8.

12. Ivashkiv LB, Hu X. Signaling by STATs. Arthritis Res Ther. 2004; 6: 159-68.

13. O'Shea JJ, Schwartz DM, Villarino AV, et al. The JAK-STAT pathway: impact on human disease and therapeutic intervention*. AnnuRev Med. 2015; 66: 311-28.

14. Fan Y, Mao R, Yang J. NF-кB and STAT3 signaling pathways collaboratively link inflammation to cancer. Prot Cell. 2013; 4: 176-85.

15. Zare F, Dehghan-Manshadi M, Mirshafiey A. The signal transducer and activator of transcription factors lodge in immunopathogene- 
sis of rheumatoid arthritis. Reumatismo. 2015; 67: 127-37.

16. O'Shea JJ, Lahesmaa R, Vahedi G, et al. Genomic views of STAT function in CD4+ T helper cell differentiation. Nat Rev Immunol. 2011; 11: 239-50.

17. Oppmann B, Lesley R, Blom B, et al. Novel p19 protein engages IL-12p40 to form a cytokine, IL-23, with biological activities similar as well as distinct from IL-12. Immunity. 2000; 13: 715-25.

18. Farrar JD, Smith JD, Murphy TL, et al. Selective loss of type I interferon-induced STAT4 activation caused by a minisatellite insertion in mouse Stat2. Nat Immunol. 2000; 1: 65-9.

19. Liang Y, Pan H-F, Ye D-Q. Therapeutic potential of STAT4 in autoimmunity. Expert Opin Ther Targets. 2014; 18: 945-60.

20. O’Malley JT, Sehra S, Thieu VT, et al. Signal transducer and activator of transcription $4 \mathrm{lim}$ its the development of adaptive regulatory $\mathrm{T}$ cells. Immunology. 2009; 127: 587-95.

21. De Herve M-gDG, Durali D, Dembele B, et al. Interferon-alpha triggers B cell effector $1(\mathrm{Be} 1)$ commitment. PLoS One. 2011; 6: e19366-e.

22. Li Q, Eppolito C, Odunsi K, Shrikant PA. IL12-programmed long-term $\mathrm{CD} 8+\mathrm{T}$ cell responses require STAT4. J Immunol. 2006; 177: 7618-25.

23. Mathur AN, Chang H-C, Zisoulis DG, et al. STAT3 and STAT4 direct development of IL17-secreting Th cells. J Immunol. 2007; 178: 4901-7.

24. Nishikomori R, Usui T, Wu C-Y, et al. Activated STAT4 has an essential role in Th1 differentiation and proliferation that is independent of its role in the maintenance of IL-12R $\beta 2$ chain expression and signaling. J Immunol. 2002; 169: 4388-98.

25. Bathon JM, Martin RW, Fleischmann RM, et al. A comparison of etanercept and methotrexate in patients with early rheumatoid arthritis. N Engl J Med. 2000; 343: 1586Đ93.

26. Maini RN, Breedveld FC, Kalden JR, et al. Therapeutic efP cacy of multiple intravenous infusions of anti-tumor necrosis factor alpha monoclonal antibody combined with low-dose weekly methotrexate in rheumatoid arthritis. Arthritis Rheum. 1998; 41: $1552 \nsupseteq 63$

27. Saag KG, Teng GG, Patkar NM, et al. American College of Rheumatology 2008 recommendations for the use of nonbiologic and biologic diseasemodifying antirheumatic drugs in rheumatoid arthritis. Arthritis Rheum. 2008; 59: 762-84.

28. Mathias SD, Colwell HH, Miller DP, et al. Health-related quality of life and functional status of patients with rheumatoid arthritis randomly assigned to receive etanercept or placebo. Clin Ther. 2000; 22: 128-39.

29. Moreland LW, Schiff MH, Baumgartner SW, et al. Etanercept therapy in rheumatoid arthritis: a randomized, controlled trial. Ann Intern Med. 1999; 130: 478-86.

30. van de Putte LB, Atkins C, Malaise M, et al. Efficacy and safety of adalimumab as monotherapy in patients with rheumatoid arthritis for whom previous disease modifying antirheumatic drug treatment has failed. Ann Rheum Dis. 2004; 63: 508-16.

31. Nikiphorou E, Negoescu A, Fitzpatrick JD, et al. Indispensable or intolerable? Methotrexate in patients with rheumatoid and psoriatic arthritis: a retrospective review of discontinuation rates from a large UK cohort. Clin Rheumatol. 2014; 33: 609-14.

32. Canadian Agency for Drugs and Technologies in Health. Clinical Review Report: Baricitinib (Olumiant): (Eli Lilly Canada Inc.): Indication: For use in combination with methotrexate (MTX) for the treatment of adult patients with moderate to severe rheumatoid arthritis who have responded inadequately to one or more disease-modifying antirheumatic drugs (DMARDs). Baricitinib may also be used as monotherapy in cases of intolerance to MTX [Internet]. this work is distributed under the terms of a Creative Commons AttributionNonCommercial-NoDerivatives 4.0 International licence (CC BY-NC-ND), a copy of which is available at http://creativecommons. org/licenses/by-nc-nd/4.0/ Bookshelf ID: NBK549715.

33. Fattahi MJ, Abdollahi M, Agha Mohammadi A, et al. Preclinical assessment of $\beta$-D-mannuronic acid (M2000) as a nonsteroidal anti-inflammatory drug, Immunopharmacol. Immunotoxicol. 2015; 37: 535-40.

34. Mirshafiey A, Rehm B, Sotoude M, et al. Therapeutic approach by a novel designed anti-inflammatory drug, M2000, in experimental immune complex glomerulonephritis, Immunopharmacol. Immunotoxicol. 2007; 29: 49-61.

35. Mirshafiey A, Rehm B, Abhari RS, et al. Production of M2000 (beta-D-mannuronic acid) and its therapeutic effect on experimental nephritis. Environ Toxicol Pharmacol. 2007; 24: 60-6.

36. Mirshafiey A, Cuzzocrea S, Rehm B, Matsuo H. M2000: a revolution in pharmacology. Med Sci Monit. 2005; 11; 153-63.

37. Mirshafiey A, Rehm BH, Sahmani AA, et al. M-2000, as a new anti-inflammatory molecule in treatment of experimental nephritis, Immunopharmacol. Immunotoxicol. 2004; 26: 611-9.

38. Ahmadi H, Jamshidi AR, Gharibdoost F, et al. A phase I/II randomized, controlled, clinical trial for assessment of the efficacy and safety of $\beta$-Dmannuronic acid in rheumatoid arthritis patients. Inflammopharmacology. 2018; 26: 737-45.

39. Rezaieyazdi Z, Farooqi A, Soleymani-Salehabadi $\mathrm{H}$, et al. International multicenter rand- 
omized, placebo-controlled phase III clinical trial of $\beta$-d-mannuronic acid in rheumatoid arthritis patients. Inflammopharmacology. 2018 [Epub ahead of print].

40. Mirshafiey A, Cuzzocrea S, Rehm B, et al. Treatment of experimental arthritis with M2000, a novel designed non-steroidal antiinflammatory drug. Scand J Immunol. 2005; 61: 435-41.

41. Mirshafiey A, Cuzzocrea S, Rehm BH, Matsuo H. M2000: a revolution in pharmacology. Med Sci Monit. 2005; 11: Pi53-Pi63.

42. Mirshafiey A, Matsuo H, Nakane S, et al. Novel immunosuppressive therapy by M2000 in experimental multiple sclerosis. Immunopharmacol Immunotoxicol. 2005; 27: 255-65.

43. Mirshafiey A, Rehm B, Cuzzocrea S, et al. M2000, foundation of a new generation among NSAIDs. Lett Drug Des Discov. 2005; 2: 412-23.

44. Lipsky PE. Are new agents needed to treat RA? Nat Rev Rheumatol. 2009; 5: 521-2.

45. van Steenbergen HW, da Silva JAP, Huizinga TWJ, van der Helm-van Mil AHM. Preventing progression from arthralgia to arthritis: targeting the right patients. Nature reviews. Rheumatology. 2005; 14: 32-41,
46. Buckley F, Finckh A, Huizinga TWJ, et al. Comparative Efficacy of Novel DMARDs as Monotherapy and in Combination with Methotrexate in Rheumatoid Arthritis Patients with Inadequate Response to Conventional DMARDs: A Network Meta-Analysis. J Manag Care Spec Pharm. 2015; 21: 409-23.

47. Wang F, Sengupta TK, Zhong Z, Ivashkiv LB. Regulation of the balance of cytokine production and the signal transducer and activator of transcription (STAT) transcription factor activity by cytokines and inflammatory synovial fluids. J Exp Med. 1995; 182: 1825-31.

48. Shouda T, Yoshida T, Hanada T, et al. Induction of the cytokine signal regulator SOCS3/ CIS3 as a therapeutic strategy for treating inflammatory arthritis. J Clin Invest. 2001; 108: 1781-8.

49. Kasperkovitz PV, Verbeet NL, Smeets TJ, et al. Activation of the STAT1 pathway in rheumatoid arthritis. Ann Rheum Dis. 2004; 63: 233-9.

50. Jones GW, Greenhill CJ, Williams JO, et al. Exacerbated inflammatory arthritis in response to hyperactive gp130 signalling isindependent of IL-17A. Ann Rheum Dis. 2013; 72: 1738-42. 